\title{
ROBUST APPROACH TOWARDS CONTEXT DEPENDANT INFORMATION SHARING IN DISTRIBUTED ENVIRONMENTS
}

\author{
Jenny Lundberg, Rune Gustavsson \\ Department of Interaction and Systems Design, Blekinge Institute of Technology, 37225 Ronneby, Sweden \\ jlu@bth.se,rgu@bth.se
}

Keywords: Information sharing in teams, semantics, context, abbreviation, robustness

\begin{abstract}
In the paper we propose a robust approach towards context dependant information modelling supporting trustworthy information exchange. Shortcomings and challenges of present approaches of syntax-based information modelling in dynamic context are identified. Basic principles are introduced and used to provide a robust approach towards meeting some of those challenges. The approach has a main aim of reducing brittleness of context dependant information and enabling intelligible information handling in distributed environments. The application domain is Emergency Service Centres, where the distributed handling of emergency calls in life critical situations of future change is in focus. The main contribution in the paper is a principled approach of use of abbreviations in dynamic emergency situations. Points of interaction for coordination are introduced as a tool supporting mappings of abbreviations between different contexts.
\end{abstract}

\section{INTRODUCTION}

Design, implementation and maintenance of digital support for handling of tasks in life-critical situations are a challenge. We have addressed some of those challenges even for distributed organizations such as Emergency Service Centres (Lundberg, 2007). The operators handling emergency calls rely on proper ICT systems supporting their everyday work. In this life-critical context abbreviations are commonly used mainly as a way of saving time, but also as a quality assurance method by introducing structured actiontypes related to calls in a semantically unambiguous way.

Abbreviation-based information exchange is common in many life-critical situations such as dealing with emergency calls (the main application of this paper), Air Traffic Control (ATC), operator control of critical infrastructures, and in several medical applications. There are some clear benefits of abbreviation-based information sharing in teams but also some inherit, and potentially catastrophic, limitations of this approach. In the following Section 2 Setting the scene, we illustrate those aspects and identify some challenges towards ensuring semantically correct context dependent information exchange in teams. The reminder of this paper is organized as follows. Section 3 present our robust abbreviation approach based on changes of context with a specific focus upon information modelling, common ground, coordination, situations and work-flows. Other approaches are shortly described in Section 4. In Section 5 we revisit our challenges of Section 2 and summarise our approach with some pointers of future research. The paper ends with references, presented in Section 6.

\section{SETTING THE SCENE}

To illustrate the challenges addressed in the paper, we introduce a well-known example of using abbreviation-based reasoning. That is 
reasoning based on results done by handheld calculators as depicted in the following Figure 1.

The result obtained by the calculator is the depicted numeric value 1.41421356237095 . This value is calculated using the displayed equation including ordinary numeric calculations and the value of $\cos (\mathrm{pi})$ and sqrt(2).

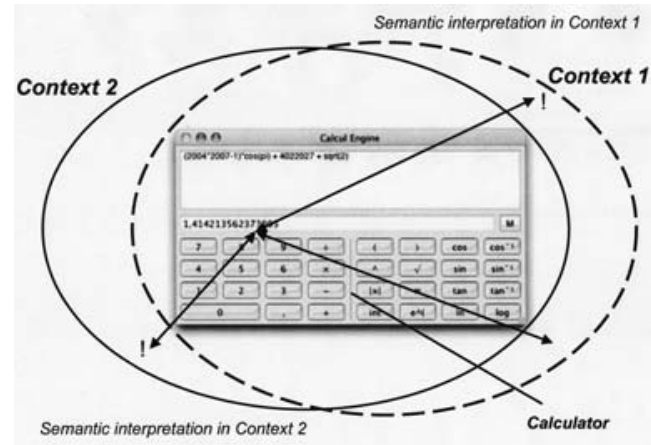

Figure 1: Reasoning based on support by calculators

The semantic information (value) can, however, only be assessed by the user of the Calcul Engine in the given context. Different contexts typically entail different semantic information from the same calculated value. In fact, this example illustrates the power of algorithmic numerical calculation where the syntax determines the semantics of the abbreviations used, i.e., numbers, numerical operators, and numeric functions. The power of algorithmic calculations is that they are context free and the interpretation of the results, by the users, is separated from the calculations of numbers. In fact, the power of mathematical models (reuse in modelling different applications) is due to their independence of semantic meaning!

To further illustrate the strengths and weaknesses of using abbreviation based reasoning, we now take a closer look at workflow support in Emergency Service Centers (ESC) and Air Traffic Control Centers (ATC).

The workflow within individual ESC can be described as a sequence of states.

- State 1: Classification of incoming calls

- State 2: Identification of appropriate actions.

- $\quad$ State 3: Deployment of teams

- $\quad$ State 4: Debriefing and reporting

The tasks of each state are governed by a set of abbreviations. The operators within the individual centers understand how to faithfully code and decode these abbreviations due to continuous training and evaluation. Abbreviations support efficient quality assured workflow support with possibilities of parallelisms between states. That is quality and efficiency.

The power of this type of abbreviations is also a weakness. Inherent in the power of abbreviations is a stable set of action-types corresponding to the abbreviations and a closed group of users to enable efficient training and other means of quality assurance of the shared intended meaning of the abbreviations. When connecting geographically separated centers, and/or changing tasks to enable more dynamic call centre handling, the mechanisms of abbreviations becomes an obstacle against changes and hence an issue to consider in depth (Lundberg, 2007). In short, how could we, in a principled way, handle context changes in abbreviation based information exchange?

The following example illustrates the critical dependency on a common understanding of context-dependencies in abbreviation-based information exchange. The example of misunderstand-ding in coordination is due to the fact that the two main actors involved didn't succeed in handling two implicit different contexts with overlapping abbreviations.

In the example, an air traffic controller and a pilot misunderstood the meaning of the abbreviation 'holding'. In December 1995, the disaster of American Airlines Flight 965 from Miami to Columbia, resulted in a loss of 159 human lives as a direct consequence to this misunderstanding. Part of the conversation was as follows, where the air traffic controller asked the pilot;

\section{- Are you holding? \\ The pilot confirms with: \\ - Roger, we're holding.}

They misunderstood each other due to the unsuccessful establishment of an agreed-upon meaning of the word "holding". Did it mean holding latitude or holding rate of decent? The air traffic controller and the pilot had different contexts and interpretations of what 'holding' meant. As the air traffic controller understood the holding as to holding latitude, the pilot understood it as holding rate of decent. A closer focus upon the situation, and the fact that they had two different contexts could most probably have avoided the loss of lives. 
Those two examples illustrate the following two inherent weaknesses with abbreviation based information sharing:

- $\quad$ Problems associated with changes of contexts

- $\quad$ Problems in understanding and discovery of implicit sharing of abbreviations in different contexts

The following Figure 2 captures the semantic hurdles of mapping of intentions between a sender and a receiver.

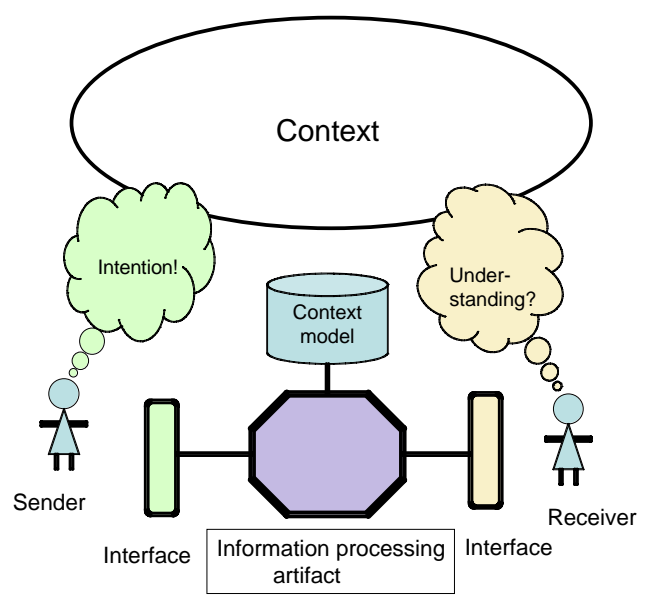

Figure 2. Semantic hurdles in semantically correct mappings between sender and receiver

We have highlighted in the figure the challenges related to introduction of an information-processing artifact between the sender and receiver. The face-to-face communication in natural language has thus been disrupted with a processing unit with two interfaces and a context model (CM) used by the processing unit to perform syntax based (rule-based) translation between the input format at the sender interface and the output format at the receiver interface. In Figure 1 the Context Model is the numeric calculation algorithms of Calcul Engine.

In short, how can we model and trustworthy convey meaning of artifact-mediated information in distributed environments when change is the rule and not the exception?

The essence of this challenge is addressing the semantically correct mapping of the intention of the information (by the sender) to the proper actions of the receiver. This challenge has been in focus of researchers in the fields of natural languages, cognitive science, Artificial Intelligence, Cognitive systems engineering, Knowledge intensive systems engineering and HMI engineering since decades.

In the case of abbreviations the sender and receiver agree about the semantic meaning of the abbreviation and the tasks it should and could/could not handle. Furthermore, the contextual meaning of the information at hand has been reduced to the syntax of the abbreviation. The remaining part of the context is shared interpretation of the abbreviation between sender and receiver as illustrated in using hand held calculators (Figure 1).

As we have earlier noted; in certain contexts, such as in numerical calculations, the Context model (CM) of Figure 2 could indeed be purely syntax based, i.e., the syntax of numerical calculations fulfills the needs we have on the context model whenever we need to do numerical calculations! In short, numerical calculations in any context obey the same syntax based rules. In fact, numerical calculations are examples of algorithmic applications where the reasoning power of the computational artifact is syntax oriented and the interpretation of the relevance of the computations in a given context lies in the agreed upon modeling principles and interpretation by the sender and receiver at the two interfaces of Figure 2.

However, turning to knowledge-intensive applications, such as support systems in real-world decision-making, the situation is fundamentally different. Let envelope, $\operatorname{Env}(\mathrm{CM})$, denote the set of computations enabled by the Information processing artifact in Figure 2, given the Context model CM. Let $\operatorname{Comp}(\mathrm{C})$, denote the set of desirable computations by the actors of Figure 2 . Clearly we always have that $\operatorname{Env}(\mathrm{CM})$ is a subset of Comp(C). In the examples of abbreviations and algorithmic application we can cope with this difference by adding agreed-upon context dependant semantic interpretation to the computational results. In general this is not the case for knowledge-intensive applications due to the (unintended) change of context. The difference between Env(CM) and Comp is sometime denoted the brittleness of the CM model.

Despite this inherent shortcoming of machine readable context models there have been considerable efforts devoted to formal models and ontologies of different domains. An ontology is a syntactic specification of concepts and their relations in a given domain. An ontology defines a formal semantic of a domain. Large amounts of 
resources have been spent on efforts creation of, for instance, enterprise ontologies.

The purpose of having a shared or at least intelligible enterprise wide semantic is to enable an understanding of the business both within the company as well as with customers and businessto-business. However, this approach has not produced the intended success (Hepp, 2007). One of the main reasons for this back-lash is due to the lack of understanding how changes in business processes entails proper and controlled changes in the corresponding $\mathrm{CM}$ as well as the specifications and changes of the interfaces of Figure 2. For enterprises in development and change, a control of ontological framework and support for change of contexts and interfaces is crucial for success. Our Robust abbreviation-based approach supporting change (Section 3) gives some pointers to those ends.

\section{ROBUST ABBREVIATION- BASED CHANGES OF CONTEXT}

As illustrated in our examples of ESC and ATC in Section 2 abbreviations can be seen as compiled support of context dependent workflows. The compiled version of workflows is sets of states. In order to control and support changes of abbreviation-based support we need to have a more abstract model of states, that is situations, and information flows to support robust changes of Context Models, Interfaces, and Contexts (Figure 2).

The remaining part of this section is thus addressing the following issues:

Section 3.1 Information modelling

Section 3.2 Common ground and coordination

Section 3.3 Situations and workflows

Section 3.4 Our approach of robust change

\subsection{Information modelling}

Keith Devlin (Devlin, 2001) has provided a semantic based logical framework supporting understanding and structuring of information (InfoSense). The logical framework has been influenced by the work of Barwise and Perry (Barwise and Perry, 1999) at Stanford in the 1980's. They developed their theories in order to understand human languages as communication of meaning, semantics and pragmatics. Suitable adaptations of the theories will provide us with models and techniques to address types of situations and hence workflows (Brandt, 2007; Östlund, 2007).

Devlin's logical framework in structuring of information can preferably be seen as a high level description of information exchange. The connection between Information (understood, or interpreted, by a human agent) and its Representation is captured by the following equation:

$$
\text { Information }=\text { Representation }+ \text { Interpretation }
$$

The equation describe that information are visible via a representation. The representation could for example be a book, a computer system or similar. The Interpretation describes the interpretation capabilities of the receiving agent. As an example, we have a situation of a fire, and a rescue person sees smoke. The rescue person makes the general assumption that there is a fire, since smoke implies fire. Thus the constraint of the rescue person's knowledge about smoke and fire makes him understand that this 'type' of seeing smoke, are related to the 'situation' fire. One of Devlin's basic contributions in InfoSense is to clarify the relations between representations and the proper interpretations by users to identify the intended situations (contexts).

The exchange of information between a sender and receiver can be described as follows (Figure 2):

The sender, in figure 2, wants to inform the receiver of a Situation S. The Representation of the situation is described by a sequence of abbreviations $A_{s}$ that is fed into the Sender interface of the artifact. The sequence $A_{s}$ is processed by the $\mathrm{CM}$ and produces a output sequence of abbreviations $A_{r}$. The receiver interprets $A_{r}$ and can infer the Situation $\mathrm{S}$. If we assume that the syntax based processing is correct and $\mathrm{A}_{\mathrm{s}}$ and $\mathrm{A}_{\mathrm{r}}$ have agreed upon semantics then the sender has successfully informed the receiver about the situation S and proper actions can be taken. Agreed upon semantics of situations are denoted common grounds (Devlin, 2001).

\subsection{Common ground and coordination}

Common ground between stakeholders thus enables correct abbreviation based semantic information exchange related to situations. In abbreviation based information exchange as in our 
examples ESC and ATC the common ground is the agreed upon interpretation of sets of abbreviations. Trusted coordination in those teams can thus be assured by proper training of skills mapping between situations and sequences of abbreviations. Abbreviations can thus be seen as coordination mechanisms in ESC, ATC and similar applications.

\subsection{Situations and workflows}

In Section 2 we identified that the workflow in a ESC could be identified by 4 states. These states are in fact compilations of corresponding four context dependant Situations; $\mathrm{S}_{1}, \mathrm{~S}_{2}, \mathrm{~S}_{3}$, and $\mathrm{S}_{4}$.

To enable a principled change of contexts in abbreviation based coordination a first step is to identify the corresponding set of situations that underpin the workflows at hand. These are complex tasks, not the least from a validation perspective. In our ESC case we have identified and validated a proper set of situations covering the relevant workflows. Proper methods and tools to that end include: work practise, ethnography, and situation theories (Lundberg, 2007, Brandt, 2007, Östlund, 2007, Barwise-Perry, 1999, Devlin 1991, 2001).

\subsection{Robust change of contexts}

We propose a robust approach for context dependent information modelling in critical information infrastructures. A basic information process is coordination (Chen, Sharman, Rao, Upadyaya, 2007). Coordination could be at different system levels and between different system components. In Figure 1 we model coordination at the highest system level, that is, between system actors (agent or users).

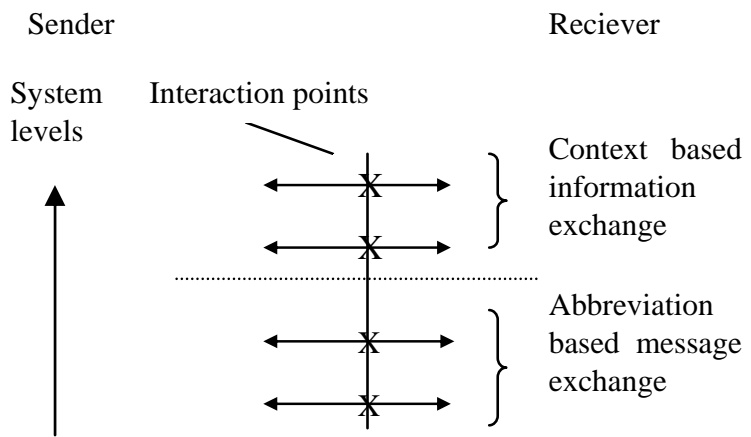

Figure 3: Interaction points between sender and receiver.
To be more specific, we introduce, in Figure 3, interaction points in dialogues between agents to enable support for different coordination aspects of Figure 2.

Overlaying the interaction points with basic information sharing of Figure 2 we recognise that the lower abbreviation based interactions, of Figure 3, are facilitated by the Context Model processing of data. The interpretation of those results is by the sender and receiver in the given context and the shared common ground supported by the abbreviations.

The main reason for introducing interaction points is that they give a natural structure of coordination between sender and receiver. Highlevel interaction points are focusing on the contextual information sharing, whence low-level interaction points are related to the information processing system. Complex coordination can be modelled using interaction points. Furthermore, interaction points capture the critical coordination challenges we have to address and maintain at the levels of common ground and processing. We also have to address the trustworthiness of translations of sequences of abbreviations between those levels.

Our model supporting robust change of contexts in abbreviation based information exchange is founded on the following steps:

1. Identify the set of situations and corresponding workflows that can be inferred from the set of abbreviations.

2. Validate mapping from situations, workflows to sequences of abbreviations

3. Describe the new context and workflows given the identified set of situations

4. Make a mapping of the new workflows on sequences of abbreviation

5. Validate mappings and introduce training of mappings among teams.

Furthermore, our approach to abbreviation based CM handling can be a basis for further investigations on causes of brittleness, and establishment of common grounds as well as offand on-line training of skills. Principled maintenance of CM due to changes of contexts is also supported. 


\section{OTHER APPROACHES}

Ongoing research and development on Web services and Semantic web are focusing on ontologies and schemas, i.e., on syntax-based structures (Hendler, 2008). Message passing between web services are facilitated by SOAP messages encoded in XML. A SOAP message between a seller and buyer could have the message:

<orderstatus>confirmed</orderstatus $>$

The issue here is to have a consistent interpretation of the abbreviation "confirmed". Again we have the problem of abbreviation-based semantics! The works on syntax-based (ontologybased) abbreviations in semantic web have the same shortcomings as abbreviations discussed in Section 2. However, those ontology-based abbreviations could be very helpful in defining the corresponding Situation-based information flows, supporting semantics in a given context, as outlined in Section 4

Approaches as (Veale, 2008) where the focus area is limited have a clear and well defined approach. However, most of the current approaches are still based in the syntax area. Our top-down approach to shared semantic based information takes a supplementary view.

\section{CHALLENGES REVISITED AND CONCLUSIONS}

In Section 1 and 2 we identified two challenges in abbreviation-based information sharing in teams:

- Change of context

- Misunderstandings

In Section 3 we outlined how we can identify the abstract Situation types and information flows in corresponding Context. A shift from localized context to a distributed one is facilitated by implementing the derived situation-based workflow and defining a new set of abbreviations to support the distributed information exchange as depicted in Figure 2.

Dealing with misunderstandings due to identical abbreviations can be solved by identifying those ambiguities using the common ground. Resolving ambiguities can be established in several ways. One way is to distinguish the different contexts by prefixes. For example; pilotholding and tower-holding in the given example presented in section 2 .

The process outlined thus establishes a robust model supporting abbreviation-based changes of contexts. It can with advantage be used in training situations as-well and to identify implicit brittleness (Nardi, O’Day, 1999).

Future work includes more elaborated models and tool supporting translation of abbreviations into rule sets and information types. Taking into account cognitive modelling and building rules from info senses constraints would be an interesting approach to consider.

\section{REFERENCES}

Barwise, J., Perry J., 1999, Situations and attitudes. CSLI Publications

Brandt, P., 2007, Information in use - aspects on information quality in workflows. BTH Dissertation series, Blekinge Institute of Technology, ISSN: 1653:2090

Chen, R., Sharman, R., Rao, H., R., Upadhyaya, S., J., 2007, Coordination in emergency response management, Communications of the ACM, vol 51, nr 5

Devlin, K., 1991, Logic and information, Cambridge University Press

Devlin, K., 2001, Info sense, W.H. Freeman and Company, New York

Hendler, J., Shadbolt, N., Hall, W., Bernes-Lee, T., and Weitzner, D., 2008, Web science: an interdisciplinary approach to understanding the web. Communications of the ACM, vol 51, no 7, 07/08

Hepp, M., 2007, Possible ontologies - how reality constrains the development of relevant ontologies, IEEE Internet comuting, published by IEEE Computer Society

Lundberg, J., 2007, Principles of workflow support in life critical situations, Blekinge Institute of Technology, ISBN/ISRN: 978-91-7295-105-1

Nardi, B. A., O’Day, V. L., 1999, Information ecologiesusing technology with heart, MIT Press

Veale, T. and Hao, Y., 2008, A context-sensitive framework for lexical ontologies. The Knowledge Engineering Review, Vol. 23:1, 101.115, Cambridge University Press.

Östlund, L., 2007, Information in use - in and out sourcing aspects on digital services. BTH Dissertation series, School of engineering, Blekinge Institute of Technology, ISSN 1653-2090, 2007 